\title{
Study of the Shape Separation of Fine Particles Using Fluid Fields
}

\section{- Dynamic Properties of Irregular Shaped Particles in Wet Cyclones $\dagger$ -}

\author{
Shigehisa Endoh, Hitoshi Ohya, Kaoru Masuda, \\ Shigeyuki Suzuki, and Hiroyuki Iwata \\ Natinal Inst. for Resources and Environ (NIRE)* \\ Chihiro Ikeda \\ Hosei Univ., **
}

\begin{abstract}
In order to apply hydrodynamic fields to the shape separation of fine particles, the dependencies of dynamic properties of particles in a cyclone on the particle shape were discussed. Glass beads, glass powders, silica sands and mica particles in a $10-100 \mu \mathrm{m}$ size range were classified into five fractions by wet cyclones. The equivalent diameters of classified particles were measured by a free settling method, and the geometric parameters were obtained from SEM microphotos. The Stokes diameters in free settling were in agreement with the mean values which were estimated for an ellipsoid and a thin disc to settle in random orientation. On the other hand, Stokes diameters in the cyclones were smaller than those in free settling. The discrepancy between these diameters was large especially for flaky mica particles, suggesting that a cyclone can be used to separate flaky particles from bulky ones.
\end{abstract}

\section{Introduction}

The uniformizing of size and shape of particles is an extremely important issue in enhancing the functions of particulate materials. Control over particle shape at the initial formation stage is desirable but with the exception of spherical particles and a few particulate materials, uniformizing of shape is difficult and moreover production costs for monodispersed regular shaped particles such as latex are extremely high, making such processes unsuitable for general powder production processes. Therefore, a shape adjusting technique after particle formation becomes indispensable in the production process and especially the development and establishment of a shape separation technique is desired.

Studies of the shape separation have centered around techniques which use the shape dependency characteristics of rolling and sliding of particles ${ }^{11}$. These techniques mainly separate particles of $500 \mu \mathrm{m}$ or more with relatively weak adhesion properties. Recently, with increasingly finer particles, separation

* 16-3 Onogawa, Tsukuba-shi Ibaraki 305, Japan

** 3-7-2 Kajino-cho, Koganei-shi, Tokyo 184, Japan

$\uparrow$ This report was originally printed in J. Soc. Powder Technology, Japan. 29, 838 (1992) in Japanese, before being translated into English with the permission of the editorial committee of the Soc. Powder Technology, Japan. techniques using electrostatic force or fluid drag can be considered to cope with such fine size areas ${ }^{2,3)}$. For example, the possibility of shape separation by rotary flow has been suggested ${ }^{4)}$ and the use of shape dependency (especially aspect ratio) ${ }^{5)}$ of particle rotation or orientation is promising. However, shape separation methods targeting fine particles remain at present yet to be established. Therefore, in the present study, experimental studies of the possibility of shape separation using rotary flow is attempted for basic studies to develop a shape separation method using a fluid field.

\section{Experiment apparatus and method}

As specimens, glass beads (Toshiba Balloty, EGB 301, abbreviated as GB), silica sand (SS), glass powder (GP) and mica particles (Nippon Mica, A100; $\mathrm{MC})$ filtered to a certain grain size range were used, Figs. 1 to 4 show the respective size distributions by dotted lines, measured by the photo extinction method using gravitational sedimentation (Shimadzu, SA (P-2). To make the conditions similar to those for classification in making measurements, particles were dispersed without any dispersing agent and only stirring in tap water by a magnetic stirrer was performed. 
Wh the classification experiment, a 5 stage type wet cyclone (Cyclosizer by Warman $\mathrm{Co}$,) was used. Its outline is shown in Fig. 5. First, a certain quantity (30 grams) of the specimen was charged in a container. Next, while tap water was being poured at a flow rate exceeding that of the classification operation condition, the specimen was gradually poured into the entire cyclones and preparatory classification was done for 5 minutes. Thereafter, the flow rate was set

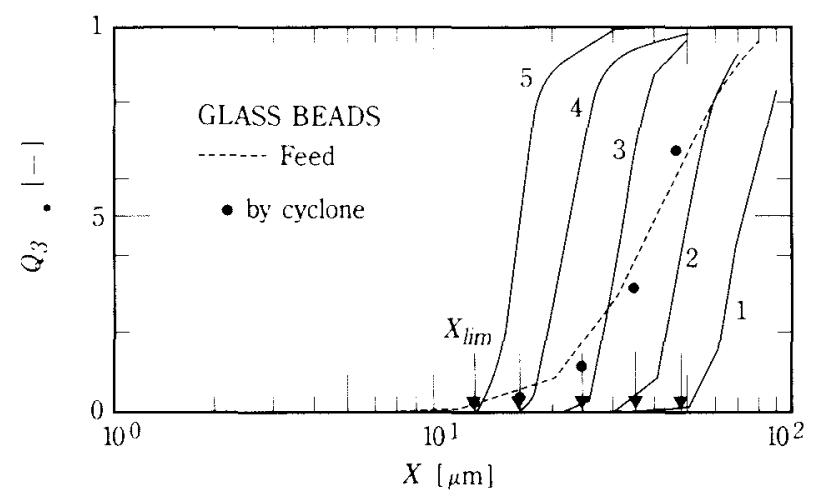

Fig. 1 Size distributions of feed particles and classified ones by the wet cyclones; Glass beads

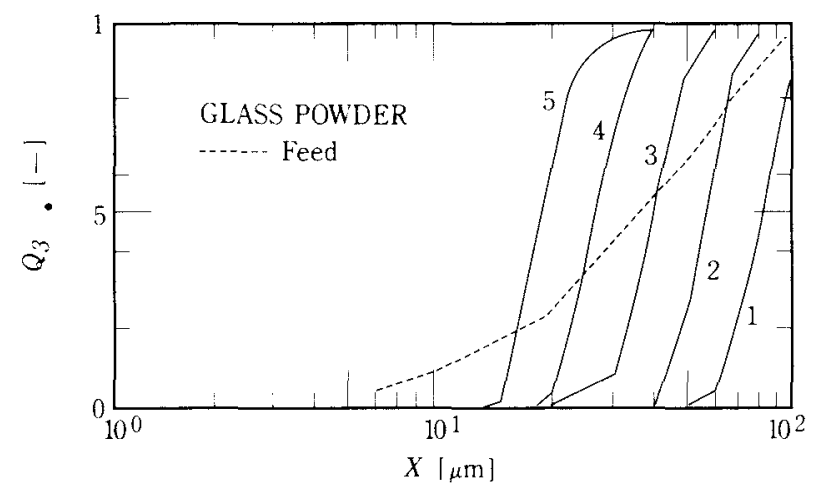

Fig. 2 Size distributions of feed particles and classified ones by the wet cyclones; Glass powder to a specified value and classification was done for 90 minutes. After classification, particles in each collector container were recovered and their particle size distribution was measured by the photo extinction sedimentation method. Also their shape was observed by a SEM. For the sake of convenience, numbers 1 to 5 were affixed from upstream to downstream of the cyclones to distinguish between the respective recovered samples.

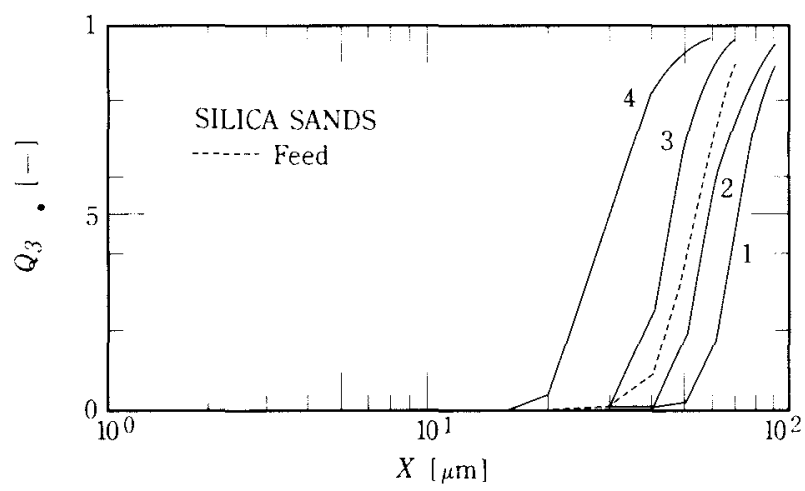

Fig. 3 Size distributions of feed particles and classified ones by the wet cyclones; Silica sand

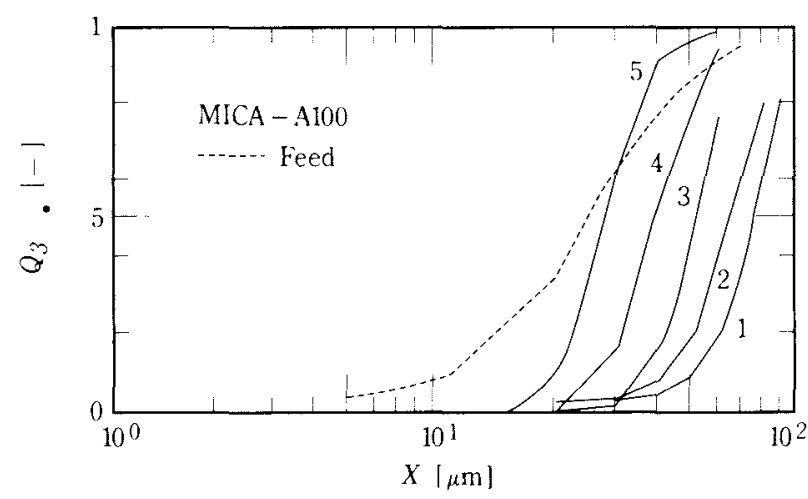

Fig. 4 Size distributions of feed particles and classified ones by the wet cyclones; Mica

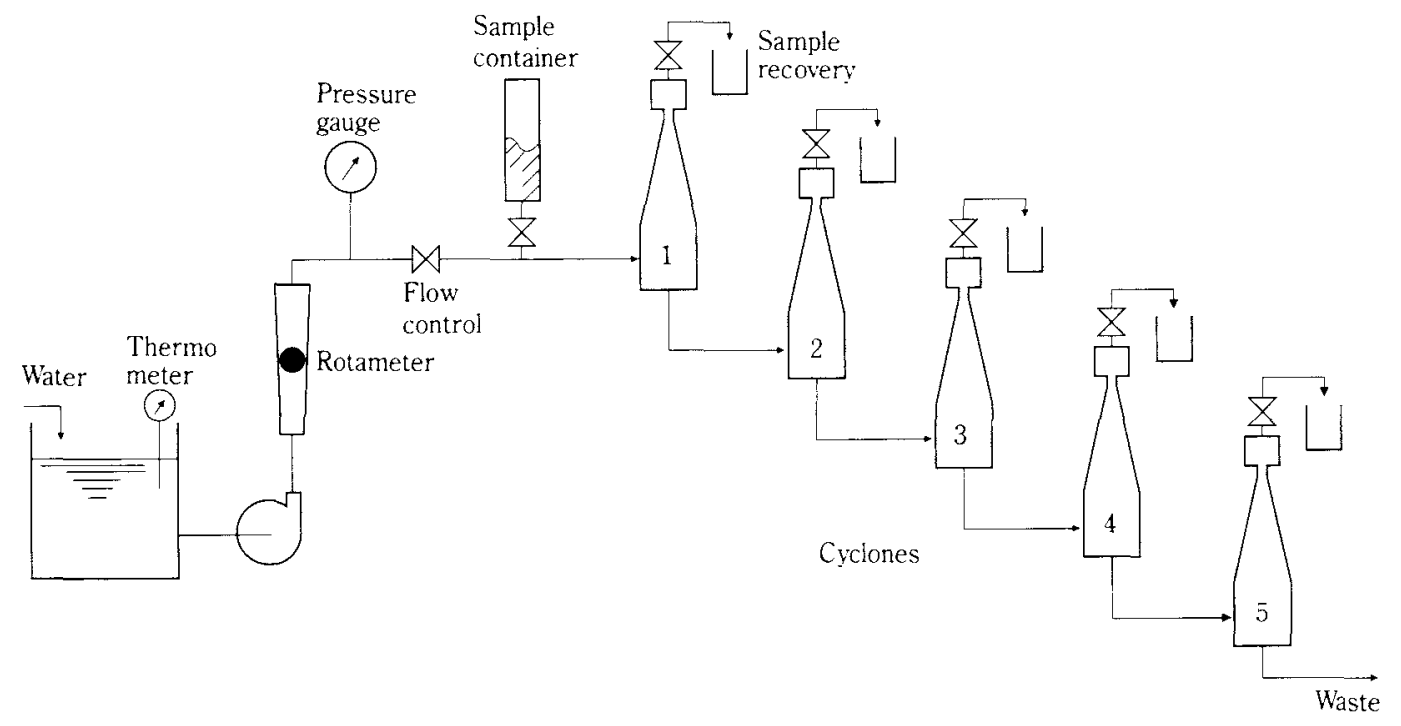

Fig. 5 Schematic diagram of experimental apparatus; CYCLOSIZER 


\section{P Experiment results and observations}

\subsection{Classification characteristics of the cyclone}

Fig. 1 shows, by solid lines, the particle size distribution of glass beads collected at each cyclone. In the Figure, the critical particle diameters $X_{\text {lim }}$ of each cyclone, specified before classification are shown by arrows. $X_{l i m}$ is determined according to the specified operation conditions ${ }^{6,7)}$ (Appendix) and in the case of the used glass beads, $X_{\text {lim }}$ was the cut off diameter of approximately $99 \%$ oversize. For comparison, the cumulative under size distribution calculated from classified particle mass was plotted against $X_{\text {lim }}$ and this roughly coincided with that of the supplied particles. Also, the geometrical standard deviation of classified particles was $1.16-1.22$, showing that an extremely strict classification was made. From the above results, it was shown that a highly accurate classification was possible with the cyclone used for this experiment and at the same time, its classification performance for spherical particles could be estimated by applying Stokes resistance rule.

Figs. 2, 3, and 4 show the size distribution of collected particles when aspherical particles were classified.

\subsection{Average particle diameters of classified particles}

Fig. 6 shows the relation between $X_{\lim }$ set for the classification operation and $50 \%$ diameter $X_{50}$ of the respective classified particles obtained by the gravitational sedimentation method. First, attention is focused on the relation of glass beads (GB). As shown in the Figure, the following relation for glass beads is obtained.

$$
X_{50}=f X_{\text {lim }}
$$

If the difference between the settling of spherical particles inside cyclones and free settling, such as the rotation of particles, are ignored, the $X_{50}$ of spherical particles can be considered as being equal to the Stokes diameter inside the cyclone. This is to say, $X_{S}$ is the Stokes Diameter inside the cyclone used in this experiment and can be considered as having the following relation with the operation parameter $X_{\text {lim }}$.

$$
X_{S}=f X_{\text {lim }} f=1.4
$$

In contrast, $X_{50}$ of the glass powder (GP) and silica sand (SS), which are aspherical, roughly satisfies the relation of Eq. (1) but the value of $f$ is somewhat larger than that for glass beads. It is conceivable that there is some difference between the free settl- ing and the settling inside the cyclone. This is to say that $X_{50}$ obtained for these particles by the gravitational sedimentation is not the Stokes diameter inside the cyclone but that at the time of free settling. Furthermore, with mica $(\mathrm{MC})$ which is flaky, $X_{50}$ of cyclones $3-5$ is fairly large compared with that of other particles. Also $X_{50}$ of cyclones 1 and 2 show roughly similar values. Obviously, it can be assumed that the behavior of particles inside the cyclone is quite different from that of free settling.

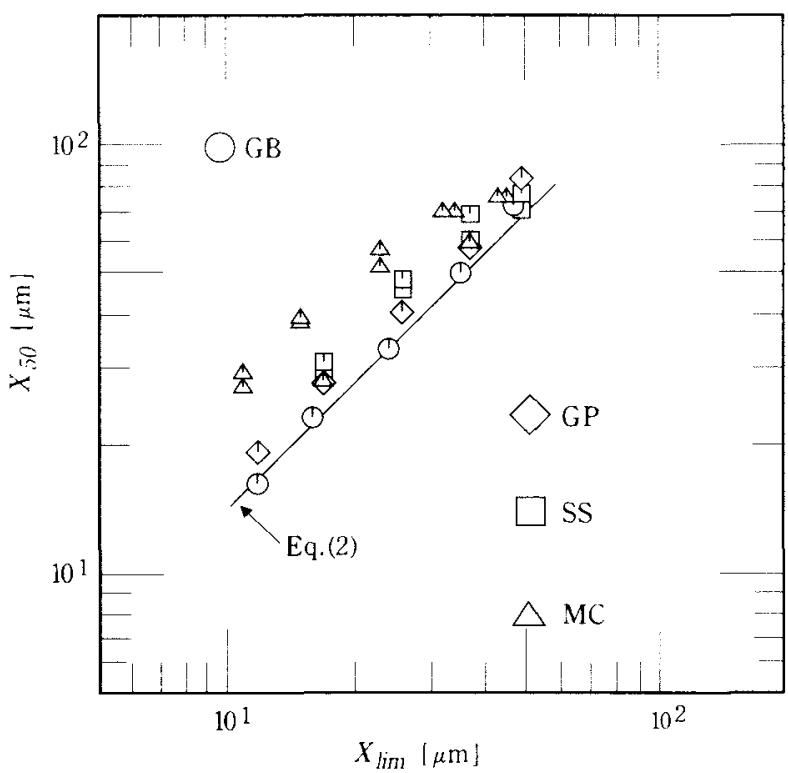

Fig. 6 Relation between cutoff diameters of the cyclones and the $50 \%$ diameters of particles classified by them

Figs 7 and 8 show SEM photos of glass powder and mica. The shape of the glass powder is massive and no characteristic change can be observed in the shape of the particles collected from upstream to downstream in the cyclones, the size being smaller but with shapes roughly similar. A similar tendency was seen in the case of silica sand. On the other hand, with mica, as seen in the photo of particles collected downstream are flaky while among the particles collected at stage 1 , many particles of massive shape were observed.

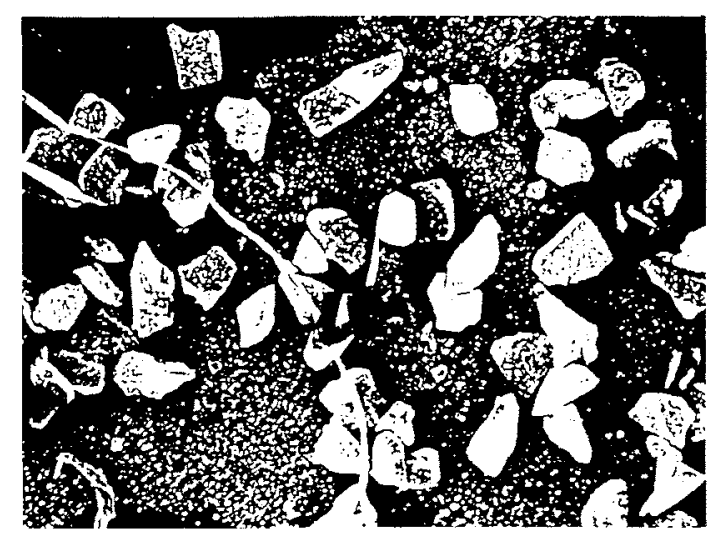

cuclone $1(x 100)$ 


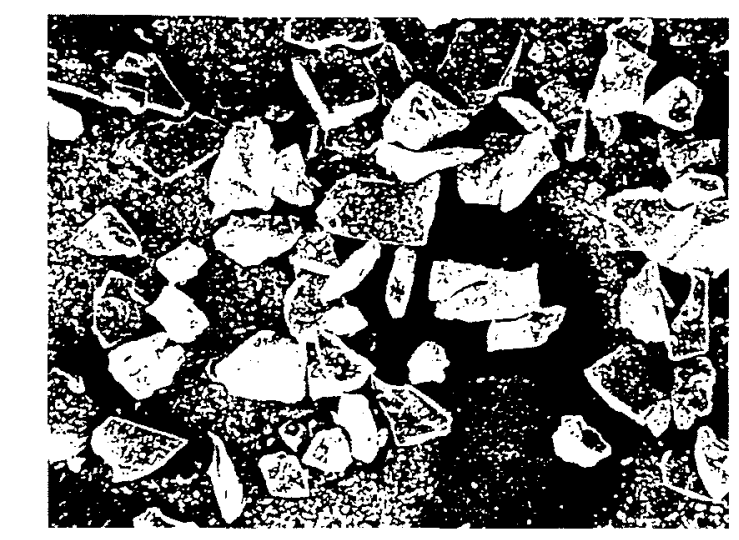

cyclone $3(\times 200)$

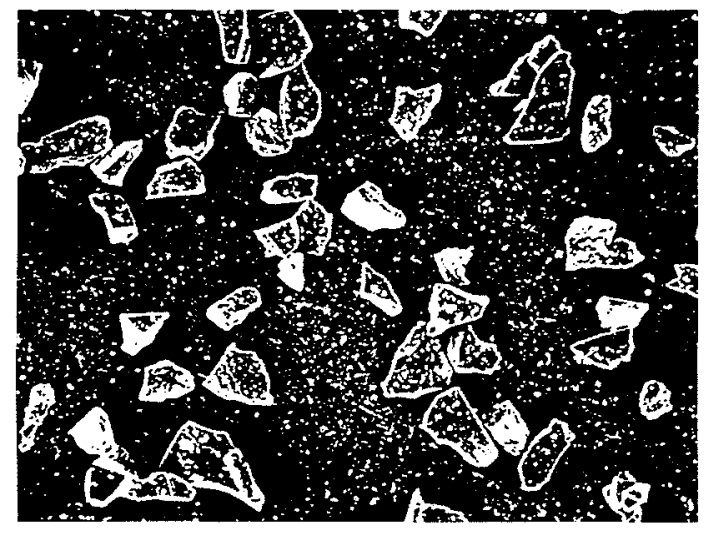

cyclone $5(\times 300)$

Fig. 7 SEM microphotos of particles classified by the cyclones; Glass powder

Fig. 9 shows the relation between Heywood diameter $X_{H}$ and Stokes diameter $X_{S} . X_{S}$ was obtained using Eq. (2) with the corresponding $X_{\text {lim }}$ and $X_{H}$ was obtained from the SEM photo taken at $0^{\circ}$ angle inclination. With massive shape silica sand and glass powder, the ratio between $X_{S}$ and $X_{H}$ is roughly unity but in contrast, $X_{H}$ of mica is generally quite larger than $X_{S}$ with the exception of stage 1 . Moreover, the $X_{H}$ in the first stage of the cyclone shows a smaller value than the values for particles collected at the 2nd and 3rd stages.Also the relation of Feret diameter to the Stokes diameter for all specimens, showed a tendency similar to the case of the Heywood diameter.

The above results contain some important implications with respect to shape classification.Firstly, as shown in Fig. 9, particles with the same value of Heywood diameter may differ in settling speed in the cyclone. This supports the possibility of making dynamic shape classification after classifying by sieving. Furthermore, according to the results shown
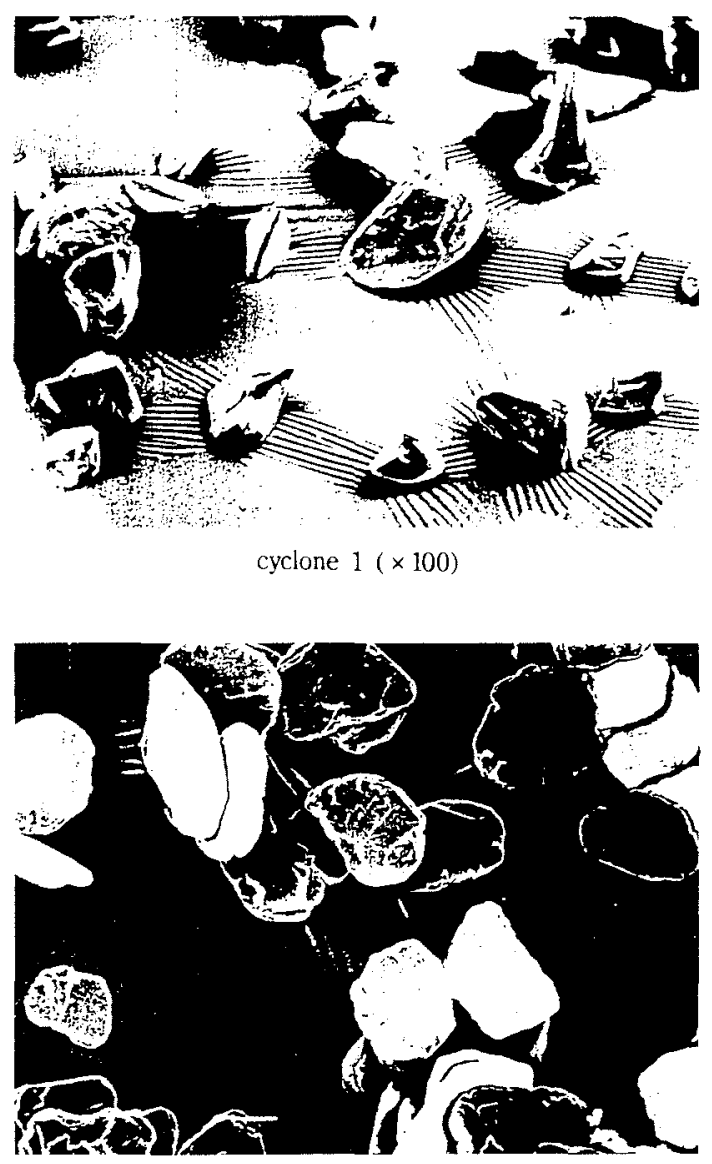

cyclone $3(\times 100)$

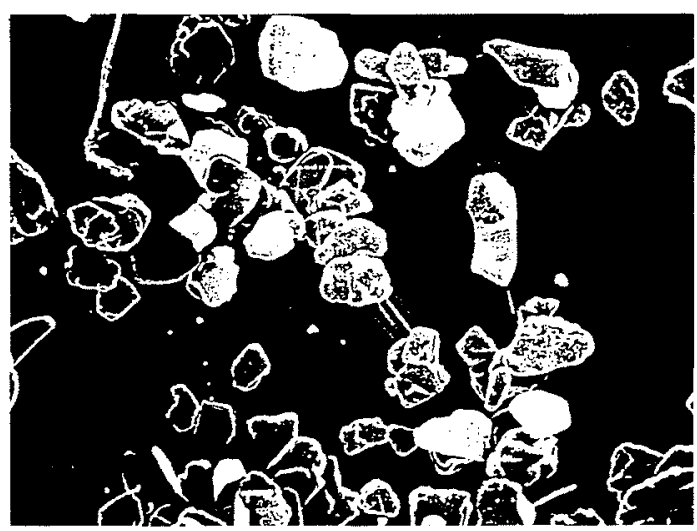

cyclone $5(\times 100)$

Fig. 8 SEM microphotos of particles classified by the cyclones; Mica

in Fig. 6, it is found that although the Stokes diameter $X_{50}$ may be the same at the time of free settling, depending on the particle, a particle may have a different sedimentation diameter $X_{S}$ in the cyclone. Also, as shown in Figs. 7, 8, and 9, a difference is clearly seen in their geometrical shapes. In other words, particle groups which have been 
gaded by gravitational sedimentation may possibly be classified further in the cyclone. For example, $X_{50}$ of mica particles which may be obtained in cyclone 1 and cyclone 2, or of mica particles which may be obtained in cyclone 3 , and of silica sand and glass powder which may be obtained in cyclone 2 may roughly be the same and therefore correct classification by gravitational sedimentation is impossible. In contrast, their $X_{S}$ differs and therefore they can be classified by cyclones due to the difference in particle shape. This is to say, the possibility of shape separation by rotary flow is implied.

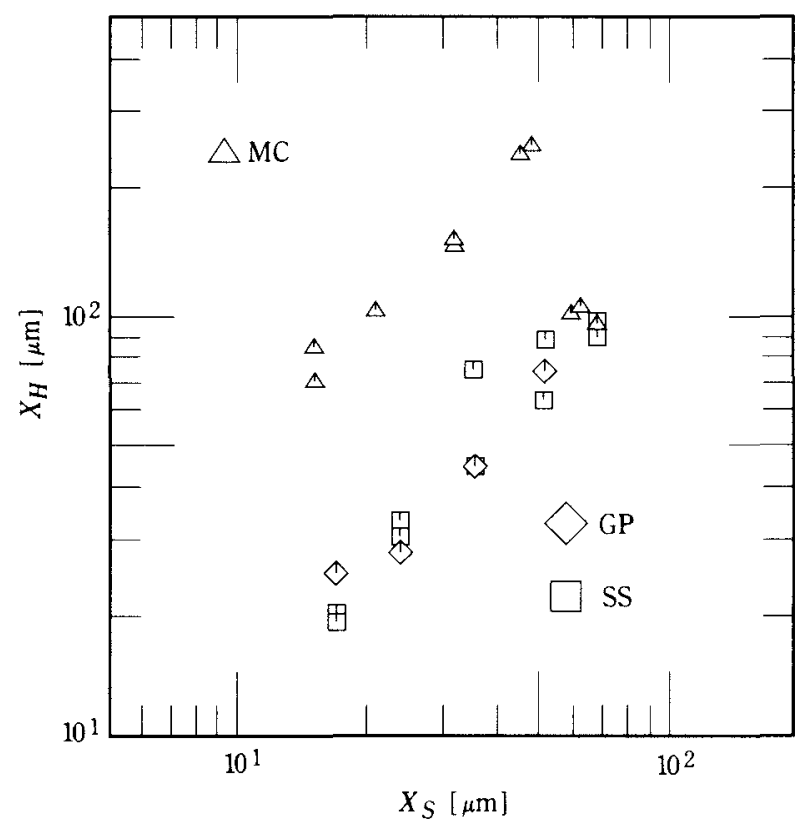

Fig. 9 Relation between Stokes diameter in the cyclones given by Eq. (2) and the Heywood diameter of particles classified by the wet cyclones

\subsection{Free settling characteristics and particle shape}

First, the case where rotating bodies having a uniform size freely settle in various orientations is studied. The settling velocity $u_{s t}$ of a particle settling in the direction of gravity with its rotating axis at an angle of $\phi$ can be obtained using the following equations ${ }^{8,9)}$,

$$
\begin{aligned}
& u_{s t}=\Delta \varrho V_{p} g K_{\phi} / 3 \pi \mu C \\
& K_{\phi}=K_{r} \sin ^{2} \phi+K_{f} \cos ^{2} \phi
\end{aligned}
$$

where, $C$ is the representative diameter, $V_{p}$ the particle volume, and $\Delta \varrho$ the density difference between the particle and fluid. Also, $K_{r}$ and $K_{f}$ are fluid resistance coefficients for various shapes and in the case of a rotating body, they are obtained using the Gans function for the particle settling in vertical and parallel to the respective rotating axis ${ }^{10,11)}$. The average value $\bar{X}_{s t}$ of Stokes diameter $X_{s t}$ is obtained using the following equation when the probability of orientation is considered,

$$
\bar{X}_{S T}=\int_{X_{\min }}^{X_{\max }} X_{s t} P\left(X_{s t}\right) d X_{s t}
$$

where, $P\left(X_{s t}\right)$ is the probability density function of $X_{s t}$ of the orientation of particles. When particles orientate randomly, $P\left(X_{s t}\right)$ satisfies the following equation.

$$
P\left(X_{s t}\right) d X_{s t}=\sin \phi d \phi
$$

First, particle settling models assuming random orientations of silica sand and glass powder are compared. For a prolate spheroid as a model particle, with $C$ to be the major axis length $2 \mathrm{a}$, the average Stokes diameter is obtained using the following equation ${ }^{12)}$,

$$
\bar{X}_{s t}=c\left[\sqrt{K_{f}}+\frac{K_{r}}{\sqrt{K_{f}-K_{r}}} \ln \frac{\sqrt{K_{f}}+\sqrt{K_{f}-K_{r}}}{\sqrt{K_{r}}}\right]
$$

where, $2 \mathrm{c}$ is the minor axis length, $K_{r}$ and $K_{f}$ are obtained using the following equations,

$$
\begin{aligned}
& K_{r}=\left[\frac{3 \zeta}{8\left(\zeta^{2}-1\right)} \frac{2 \zeta^{2}-3}{\sqrt{\zeta^{2}-1}} \ln \left\{\zeta+\sqrt{\zeta^{2}-1}\right\}+\zeta\right] \\
& K_{f}=\left[\begin{array}{lll}
\frac{3 \zeta}{\left(\zeta^{2}-1\right)} & \frac{2 \zeta^{2}-1}{\sqrt{\zeta^{2}-1}} \ln \left\{\zeta+\sqrt{\zeta^{2}-1}\right\}+\zeta
\end{array}\right]
\end{aligned}
$$

where $\zeta$ is the axis ratio a/c $(>1)$ of the spheroid. Fig. 10 shows the relation between the evaluated value $X_{\text {st, estm }}$ of Eqs. (7), (8), and (9) and the experimental value $X_{s t, \text { meas }}\left(=X_{50}\right)$. Although some dispersion is seen, no substantial discrepancy is observed and therefore it can be considered that these particles can be approximated to prolate spheroids.

Next, the case of mica is considered. Mica is obviously of a flaky shape and therefore a disk particle is considered as its model. Assumed to be a disk with $X_{H}$ as the diameter and $t$ as the thickness obtained from an image analysis, the representative diameter $C$ is set as $X_{H}$. The average diameter is obtained from Eq. (5) using the following equations. 


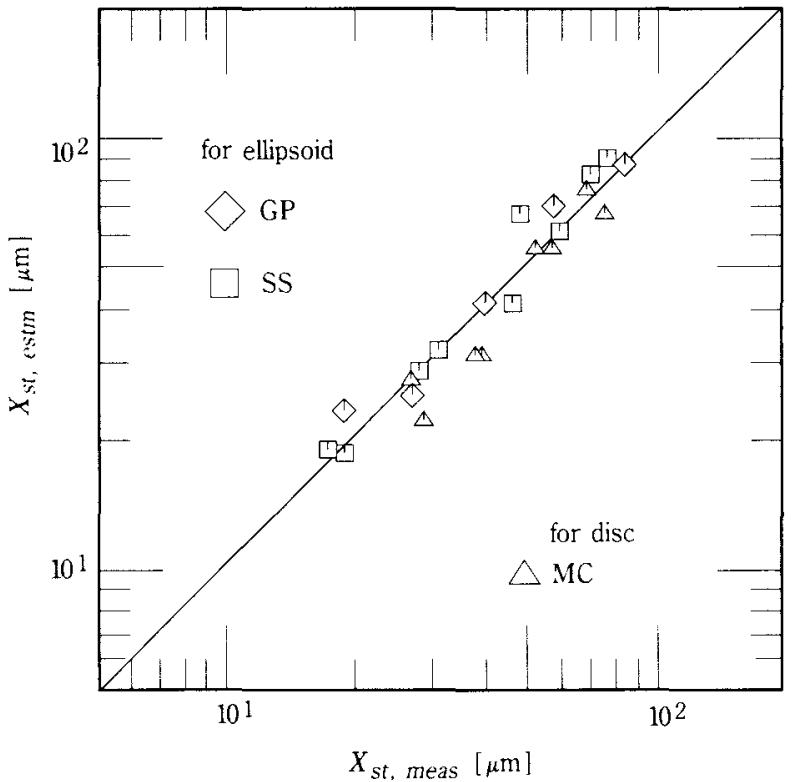

Fig. 10 Comparison of Stokes diameter obtained by photoextinction sedimentation with that estimated by Eqs. (7) and (10)

$\bar{X}_{s t}=\sqrt{\frac{3 \eta}{2}} \frac{t}{2}\left[\sqrt{K_{f}}+\frac{K_{r}}{\sqrt{K_{r}-K_{f}}}\left\{\frac{\pi}{2}-\arcsin \sqrt{K_{r} / K_{f}}\right\}\right]$

$\eta=X_{H} / t$

For $K_{r}$ and $K_{f}$, the Gans function for a disk is used. When flatness $\eta$ is constant, according to Eq. (10), $\bar{X}_{\text {st }}$ is proportional to $X_{H}$. On the other hand, when the thickness is constant, it is roughly proportional to $\sqrt{X_{H}}$. The above relations are compared in Fig. 10. Substituting the thickness obtained from a SEM photo in Eq. (10), we estimated the Stokes diameter. Since the estimated value $X_{\text {st, estm }}$ was roughly close to the experimental value, the Stokes diameter at the time of free settling of classified mica can be estimated using Eq. (10). Also, it can be considered that the orientation of flaky particles settling freely was random.

\subsection{Dynamic shape coefficient during rotary flow}

As mentioned before, since the free settling of irregular shaped particles can roughly be described by Eq. (5) which assumes random orientation, the dynamic shape factor $\varkappa_{F}$ can be obtained using the following equation,

$$
\varkappa_{F}=\left(\bar{X}_{s t} / X_{s p}\right)^{2}
$$

where $X_{s p}$ is the equal volume sphere equivalent diameter. By following this definition, the dynamic shape factor $\varkappa_{c}$ during rotary flow is expressed by the following equation.

$$
\begin{aligned}
\varkappa_{c} & =\frac{(\text { Sedimentation velocity during rotary flow) }}{\text { (Sedimentation velocity of equal volume sphere) }} \\
& =\left\{\left(X_{\text {st }} \text { during rotary flow }\right) / X_{s p}\right\}^{2}
\end{aligned}
$$

$X_{\text {st }}$ during rotary flow can be considered as $X_{\text {st }}$ obtained from Eq. (2) for an optional operation condition $X_{l i m}$. Therefore, the difference between the free settling of each particle and the settling inside the cyclone can be evaluated using the following equation.

$$
\varkappa_{c} / \varkappa_{F}=\left(X_{s} / \bar{X}_{s t}\right)^{2}
$$

Fig. 11 shows the relation between $\bar{X}_{s t}\left(=X_{s t, e s t m}\right)$ estimated with Eq. (5) and $X_{s}$. The aspect ratio $\zeta$ given by substituting the projected images of glass powder and silica sand by inertial ellipsoids is $1.1-1.3$ while the flatness $\eta$ of mica of cyclone $3-5$ was $15-25$. Therefore it is thought that the behavior of aeolotropic particle having a larger aspect ratio during rotary flow depends more strongly on its shape and that the difference with free settling becomes greater. In other words, it can be assumed that flaky particles exhibit a peculiar orientation in rotary flow and by utilizing orientation, it is believed that shape classification by rotary flow is possible.

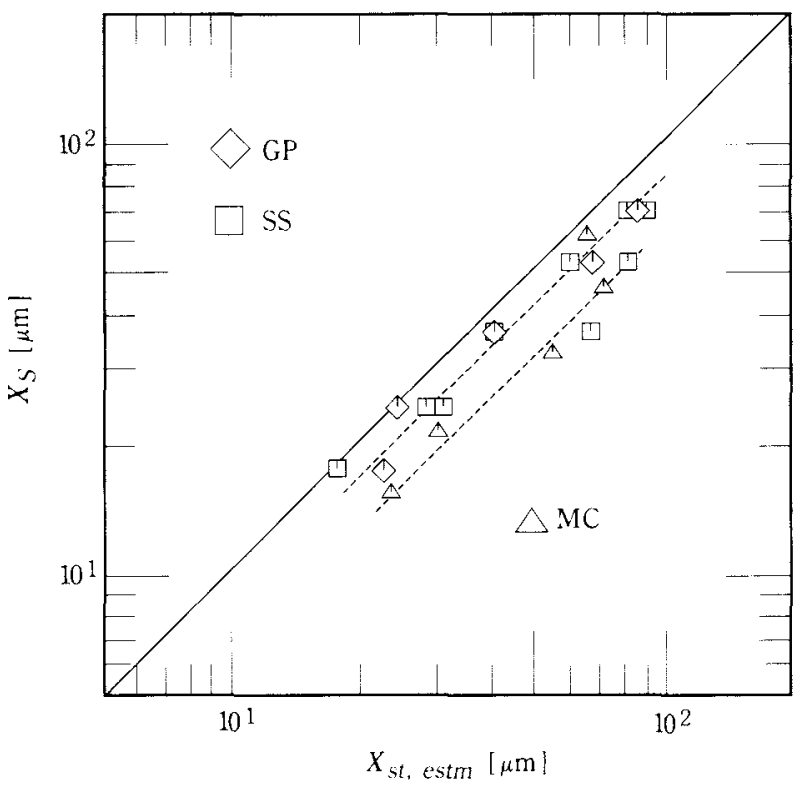

Fig. 11 Relation between Stokes diameter in the cyclones and that estimated with Eqs. (7) and (10) 
Also the following result was obtained by Kesall et al. with respect to the effect of flow rate $Q$.

$$
X=\left(Q_{0} / Q\right)^{1 / 2} X_{0}=f_{3} X_{0}
$$
particles by rotary flow, classification of aspherical particles was conducted using a wet cyclone with extremely sharp classifying characteristics and a study was made of the effect of particle shape on the dynamic behavior of particles. As a result, the following conclusions were reached.

1) The Stokes diameter of aspherical particles in free settling can be estimated by using Gans functions or dynamic shape coefficients and it is believed that the orientation at the time of free settling is random.

2) From the fact that the dynamic behavior of flaky particles in a rotary flow depends greatly on its shape compared with massive shape particles, the possibility of classification between flaky particles and massive shape particles by rotary flow is implied.

Regarding the orientation of particles inside a cyclone, further detailed clarification as necessary hereafter by observations of sedimentation.

Finally, we would like to mention that this study was made possible with the great cooperation of Mr. Koji Ishimaru, 4th year student (at the time) in the Mechanical Engineering Dept., Hosei University. Also we acknowledge the cooperation of Mr. Shinichi Itoh of the Material Resources Dept. of our institute, NIRE, in the operation of cyclones. Our gratitude to them is extended.

\section{Appendix}

The cutoff diameter $X_{0}$ of each cyclone when quartz (specific gravity 2.65) is classified under standard operation conditions $\left(20^{\circ} \mathrm{C}\right.$ water supplied at a rate of 11.6 liters/min.) is obtained based on the results ${ }^{6)}$ of Kelsall et al. ${ }^{7)}$ The general cutoff diameter $X_{\text {lim }}$ can be obtained using the following equation when fluid viscosity, particle density, flow rate and classification time are considered.

$$
X_{\lim }=f_{1} f_{2} f_{3} f_{4} X_{0}
$$

When Stokes resistance is assumed, the effects of the fluid viscosity $\mu$ and the particle fluid density difference $\Delta \varrho$ are given by:

$$
\begin{aligned}
& X=\left(\mu / \mu_{0}\right)^{1 / 2} X_{0}=f_{1} X_{0} \\
& X=\left(\Delta \varrho_{0} / \Delta \varrho\right)^{1 / 2} X_{0}=f_{2} X_{0}
\end{aligned}
$$

where, subscript 0 indicates standard conditions.

Furthermore, the effect of classification time can be obtained as follows as a function of the empirical parameter $f_{4}$.

$$
X=f_{4} X_{0}
$$

We obtain eg. (A-1) considering factors $f_{1}, f_{2}, f_{3}$ and $f_{4}$, and $X_{\text {lim }}$ can be calculated.

\section{Nomenclature}

a : large semi-axis of an ellipsoid

C : characteristic dimension of a particle

$c \quad$ : one semi-axis of an ellipsoid

$g \quad$ : gravitational acceleration $\left(\mathrm{m} / \mathrm{s}^{2}\right)$

$K_{f}, K_{r}$ : resistance coefficients along the translation axes of a particle or an ellipsoid given by Gans' function (-)

$Q_{3} \quad$ : cumulative undersize distribution (-)

$t \quad$ : thickness of a particle

$u_{s t} \quad$ : Stokes terminal velocity of a particle $(\mathrm{m} / \mathrm{s})$

$V_{p} \quad$ : volume of a particle $\left(\mathrm{m}^{3}\right)$

$X \quad$ : diameter $\quad(\mathrm{m})(\mu \mathrm{m})$

$X_{H} \quad$ : Heywood diameter obtained from projected area (m)

$X_{\text {lim }} \quad$ : cutoff diameter of a cyclone (m)

$X_{s} \quad$ : Stokes diameter inside a cyclone (m)

$X_{s p} \quad$ : equivalent spherical diameter (m)

$X_{s t} \quad$ : Stokers diameter (m)

$X_{50}: 50 \%$ diameter obtained by gravitational sedimentation

$\Delta \varrho \quad:$ density difference between a particle and fluid $\left(\mathrm{kg} / \mathrm{m}^{3}\right)$

$\zeta \quad$ : anisometry of an ellipsoid $\quad(-)$

$\eta \quad$ : flatness of a particle

$\varkappa_{F}, \varkappa_{c}:$ dynamic shape factors for free settling and settling inside a cyclone (-)

$\mu \quad$ : fluid viscosity

\section{Subscripts}

estm : estimated by using Eq. (5)

meas : measured

\section{Superscript}

- : mean 


\section{References}

1) Moro, T., H. Iwata and H. Ohya: "Report on shape separation techniques of solid particles", National Inst. for Resources and Environ. (1989)

2) Murali, C., B. Pitchumani and N. N. Clark: Int. J. Min. Pro., 18, 237 (1986)

3) Horvath, H.: J. Aerosol Sci., 10, 309 (1979)

4) Miwa, S.: "Kagaku Kogaku 2 (Ed. by J. Fujita and H. Tohata)", p. 342, Tokyo Kagaku Dojin (1972).

5) Goldsmith, H. L. and S. G. Mason: "Rheology, Theory and Application", Vol. 4, Chap. 2, Academic Press (1967)
6) Kelsall, D.F. and C.H. McAdam: Trans. Inst. Chem. Engrs., 41, 84 (1963)

7) Warman International PTY. LTD.: " CYCLOSIZER Instruction Manual" (1965)

8) Brenner, H., Chem. Eng. Sci., 18, 1 (1963)

9) Blumberg, P.N. and C.A. Mohr: AlChE J., 14 331 (1968)

10) Gans, R.: Sitzber math - physk Klasse Akad. Wiss. Munchen, 41, 191 (1911)

11) Heiss, J. H. and J. Coull: J. Chem. Eng. Progr., 48133 (1952)

12) Endoh, S., Y. Kuga and H. Iwata: Proc. Ann. Meeting of Soc. Powder Technol. Japan, p. 173 (1991) 